\title{
Cervicoplastia anterior
}

\author{
Lucas Gomes Patrocínio', José Antônio Patrocínio²
}

\section{Anterior cervicoplasty}

\author{
Palavras-chave: ritidoplastia, cervicoplastia, \\ pescoço, cirurgia plástica. \\ Key words: rythidoplasty, cervicoplasty, \\ neck, plastic surgery.
}

\section{Resumo / Summary}

\section{M}

uitos pacientes buscam correção estética da frouxidão da pele do pescoço, depósito de gordura na região submentoneana ou bandas de platisma. Em grande parte dos casos a ação medial, via cervicoplastia anterior é necessária. Objetivo: Demonstrar a casuística e avaliar os resultados e complicações com a técnica de cervicoplastia anterior no Serviço de Otorrinolaringologia da Universidade Federal de Uberlândia. Forma de estudo: Relato de série. Pacientes e Métodos: Quarenta e dois pacientes, entre 39 e 65 anos de idade, sendo $40(95,2 \%)$ do sexo feminino e $2(4,8 \%)$ do masculino, foram submetidos a cervicoplastia anterior. Retrospectivamente foram avaliados resultados e complicações. Resultados: Destes, 34 apresentaram resultados satisfatórios, 4 apresentaram déficit estético notado somente pelo cirurgião, 3 apresentaram déficit estético notado somente pelo paciente e 1 apresentou déficit estético necessitando cirurgia revisional. Ao estudo fotográfico, todos os pacientes apresentaram melhora do perfil cervical, redução das bandas de platisma e da frouxidão da pele, estabilização da musculatura cervical e acentuação do ângulo cervicomental, em graus variados. Houve complicação em 2 casos (discreto serohematoma e cicatriz um pouco alargada). Conclusão: A cervicoplastia, associada ou não à tração lateral pela ritidoplastia, é uma técnica que produz resultados satisfatórios na grande maioria dos casos.

\begin{abstract}
$\mathrm{M}$ any patients look for aesthetic correction of the laxity of neck skin, submandibular fat deposit or platisma bands. In a large part of the cases, medial action, through anterior cervicoplasty is necessary. Aim: To demonstrate the casuistic and to evaluate the results and complications with anterior cervicoplasty technique in the 0 torhinolaryngology Service of the Federal University of Uberlândia. Study design: Serie report. Patients and Methods: Forty-two patients, between 39 and 65 years of age, being 40 $(95,2 \%)$ male and $2(4,8 \%)$ female, were submitted to anterior cervicoplasty. Retrospectively, results and complications were appraised. Results: of these patients, 34 presented satisfactory results, 4 presented aesthetic deficit only noticed by the surgeon, 3 presented aesthetic deficit only noticed by the patient and 1 presented aesthetic deficit needing revisional surgery. At photographic study, all the patients presented improvement of cervical profile, reduction of platisma bands and of skin laxity, stabilization of cervical musculature, and accentuation of cervicomental angle, in various degrees. There were two complications (discreet serohematoma and little enlarged scar). Conclusion: Anterior cervicoplasty, associated or not to lateral traction, is a technique that produces satisfactory results in the great majority of the cases.
\end{abstract}

\footnotetext{
${ }^{1}$ Residente do Serviço de Otorrinolaringologia da Faculdade de Medicina da Universidade Federal de Uberlândia. 2 Professor Titular e Chefe do Serviço de O torrinolaringologia da Faculdade de Medicina da Universidade Federal de Uberlândia. Serviço de Otorrinolaringologia da Universidade Federal de Uberlândia, Uberlândia, Minas Gerais, Brasil. Endereço para correspondência: Lucas Gomes Patrocínio - Rua XV de Novembro, 327/1600 Bairro Centro Uberlândia MG $38400-214$ Tel/Fax: (0xx34) 215-1143 - E-mail: lucaspatrocinio@triang.com.br Artigo recebido em 26 de maio de 2004. Artigo aceito em 01 de julho de 2004.
} 


\section{INTRODUÇÃO}

A Ritidoplastia (lifting) ou cirurgia do rejuvenescimento facial é um procedimento que vem ganhando cada vez mais popularidade entre os otorrinolaringologistas. Depois de adquirirem capacitação em rinoplastia, seguiram em direção à pálpebra e à orelha, executando blefaroplastias e otoplastias. Agora, estão chegando ao restante da face e se iniciando nas ritidoplastias.

Trinta anos atrás, os problemas do acúmulo de gordura submentoniano e formação de bandas de platisma eram inadequadamente abordados quando do rejuvenescimento do terço inferior da face. Uma tentativa para suavizar as bandas de platisma era tensionar a pele submentoniana e cervical utilizando incisões pré-auriculares. A gordura submentoniana em excesso geralmente não era tratada. Inevitavelmente, ocorria recorrência das bandas de platisma meses após a cirurgia, principalmente nos pacientes com má elasticidade cutânea.

Por esta razão, alguns cirurgiões nos anos 60 tratavam redundância de pele submentoniana associada a ângulo cervicomandibular oblíquo com uma incisão cervical anterior direta ${ }^{1,2}$, que deixava o paciente com uma cicatriz visível. Nos anos 70, Weisman ${ }^{3}$ descreveu uma incisão submentoniana, sem excisão de pele, através da qual ele incisava as bandas do platisma e unia-as por uma zetaplastia. O utros abordavam as bandas do platisma ressecando suas margens mediais ${ }^{4}$ ou suturando suas margens laterais ou retalhos deste músculo (m.) no m. esternocleidomastóideo ${ }^{5-7}$. Algumas técnicas incorporavam a plicatura anterior das margens mediais do $\mathrm{m}$. platisma ${ }^{6,8}$. Feldman expandiu esta idéia em 1990 com o conceito da formação de um "corpete" (corset) de platisma através da sutura total das margens mediais do m. platisma ${ }^{9}$. Ramire ${ }^{10}$ modificou algumas destas técnicas descritas e introduziu novos conceitos na abordagem cervical anterior não-excisional.

Atualmente, a abordagem padrão para o rejuvenescimento da região submentoniana é uma variação da platismaplastia corset em associação a lipectomia com remoção do excesso de gordura submentoniana sob e sobre 0 m. platisma, podendo conjuntamente utilizar a liposucção.

Os avanços técnicos estão permitindo resultados estéticos impressionantes em procedimentos isolados ou associados, melhorando os resultados de cirurgias tradicionais. Descreveremos aqui a técnica de cervicoplastia anterior e nossa casuística, avaliando os resultados e complicações.

\section{PACIENTES E MÉTODOS}

\section{Pacientes}

De janeiro de 2001 a janeiro de 2004, foram realizadas 152 ritidoplastias no Serviço de Otorrinolaringologia da Universidade Federal de Uberlândia. Destes, 42 pacientes foram submetidos a cervicoplastia anterior (em 30 casos associada à tração lateral). Os pacientes apresentavam en- tre 39 e 65 anos de idade, sendo $40(95,2 \%)$ do sexo feminino e $2(4,8 \%)$ do masculino.

Todos os pacientes foram examinados e passaram por uma seleção rigorosa para serem submetidos a esta cirurgia. Foram selecionados os seguintes pacientes: jovens, com ângulo cervicomental obtuso, pescoço grosso e com gordura cervical em excesso; ou idosos, com pele elástica, pouca banda de platisma e pouca gordura cervical. A cirurgia foi contra-indicada nos pacientes com frouxidão leve, ausência de banda de platisma, ângulo cervicomental agudo, meia-idade com bandas grandes de platisma e pele sem elasticidade, idosos com bandas de platisma e dobras no pescoço, "pescoço de touro", micrognatia com indicação de cirurgia ortognática e/ ou ptose da glândula submandibular.

Os pacientes retornaram com 7, 30,60 e 180 dias de pós-operatório, sendo reavaliados quanto às possíveis complicações. Na última consulta, os pacientes foram questionados quanto à satisfação com a cirurgia e o resultado estético final foi julgado pela equipe médica do serviço.

Todos pacientes assinaram um termo de consentimento informado de acordo com a aprovação da Comissão de Ética da instituição.

\section{Técnica Cirúrgica}

A cirurgia foi executada sob anestesia local, com infiltração de lidocaína a 2\% com adrenalina 1:200.000 e leve sedação com fentanila e midazolam. 0 paciente se posicionou em decúbito dorsal com flexão posterior da cabeça. 0 cirurgião se colocou atrás da cabeça do paciente, como na cirurgia de amigdalectomia, auxiliado pela luz do foco frontal.

Com bisturi lâmina 15, realizou-se uma incisão de 3 a $4 \mathrm{~cm}$ de comprimento na dobra submental. Dissecou-se com tesoura de Metzenbaum, auxiliada pelo afastador de Aufrich, deixando um retalho subcutâneo espesso. A dissecção tem como limites a linha da mandíbula, a banda anterior do $\mathrm{m}$. esternocleidomastóideo e a cartilagem cricóide (Figura 1). Fez-se uma lipectomia sobre o $\mathrm{m}$. platisma, o m. milohióide e o m. digástrico, ressecando uma fita de gordura de $2 \times 5$ $\mathrm{cm}$ (Figura 2). Dissecou-se o m. platisma com ressecção e aproximação das bordas anteriores na linha média, da cartilagem tireóide até a sínfise da mandíbula, com sutura de Ethybond 2-0, contínua ancorada, de direção superior para inferior até a cartilagem tireóide e retornando de inferior para superior com sutura contínua simples. Após, cortou-se o m. platisma, com cautério, oblíqua e inferiormente 3 a 4 $\mathrm{cm}$ para cada lado, na borda superior da cartilagem tireóide. Suturou-se a incisão no subcutâneo com Vicryl 4-0 e a pele com sutura contínua com Prolene 5-0. 0 curativo compressivo com micropore e os pontos permaneceram por 7 dias $^{11}$.

\section{RESULTADOS}

A avaliação subjetiva final da cirurgia depende dos pontos de vista do paciente e do cirurgião, que, em alguns 
casos, diferem. D os 42 pacientes, 34 apresentaram resultados satisfatórios, 4 apresentaram déficit estético notado somente pelo cirurgião, 3 apresentaram déficit estético notado somente pelo paciente e 1 apresentou déficit estético necessitando cirurgia revisional.

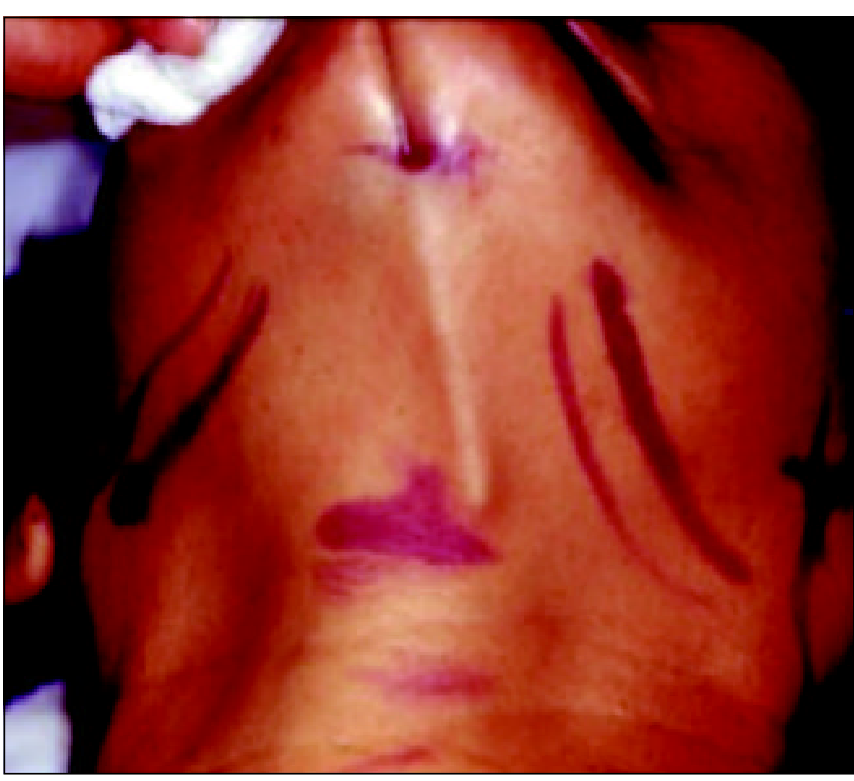

Figura 1. Fotografia demonstrando pela marcação os limites da área de elevação do retalho, o local da incisão e a infiltração do anestésico local.

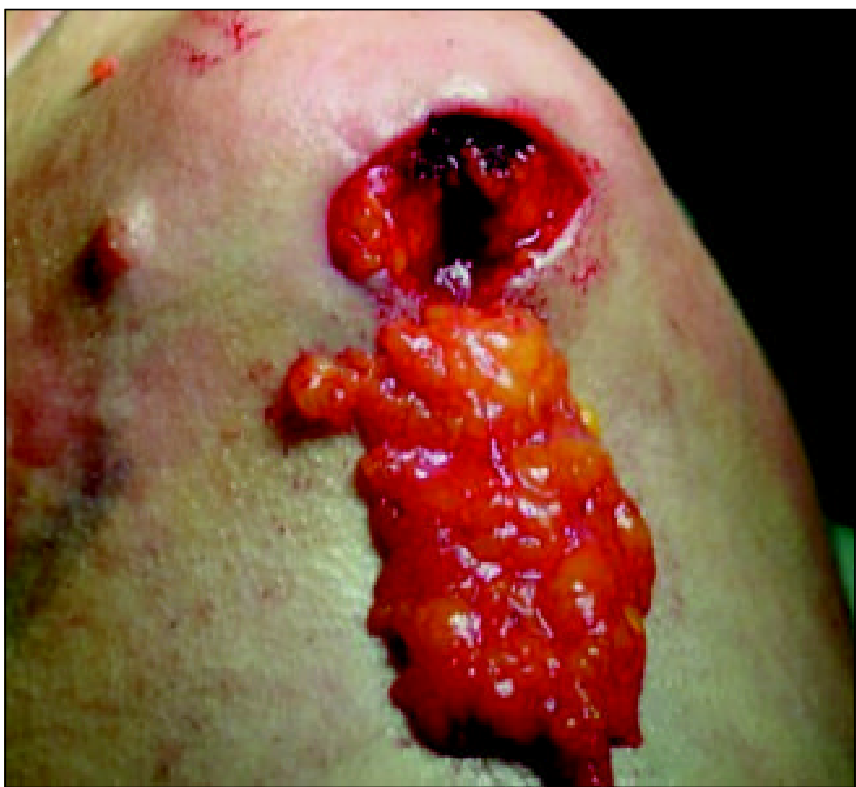

Figura 2. Fotografia demonstrando a lipectomia. Peça de $2 \times 5 \mathrm{~cm}$ da linha média.
Ao estudo fotográfico, todos os pacientes apresentaram melhora do perfil cervical, redução das bandas de platisma e da frouxidão da pele, estabilização da musculatura cervical e acentuação do ângulo cervicomental, em graus variados (Figura 3 e 4).

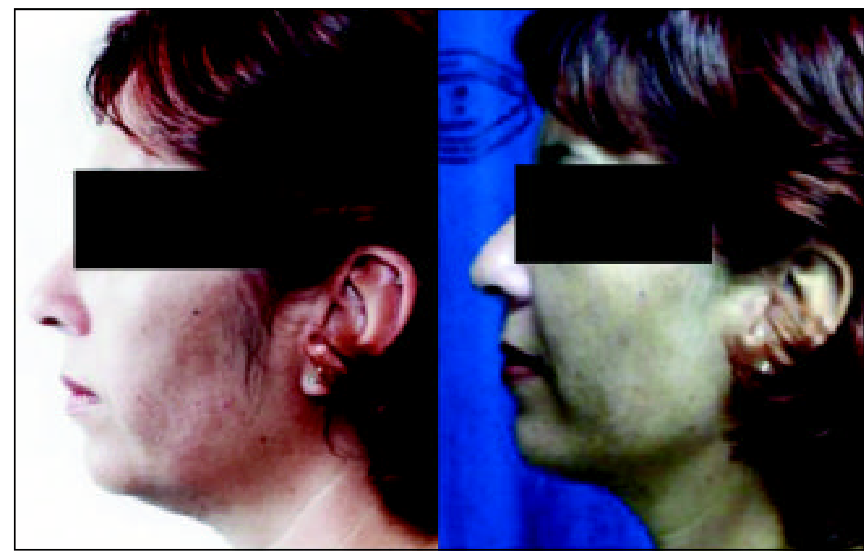

Figura 3. Visão lateral de paciente de 44 anos, feminino, com excesso de gordura submental, bandas platismais, ângulo cervicomental obtuso e frouxidão de pele. Pós-operatório (50 mês) e lateral de cervicoplastia anterior demonstrando excelente linha mandibular, melhora estética do ângulo cervicomental agudo, ausência das bandas platismais e frouxidão da pele e boa proporção do terço inferior da face.
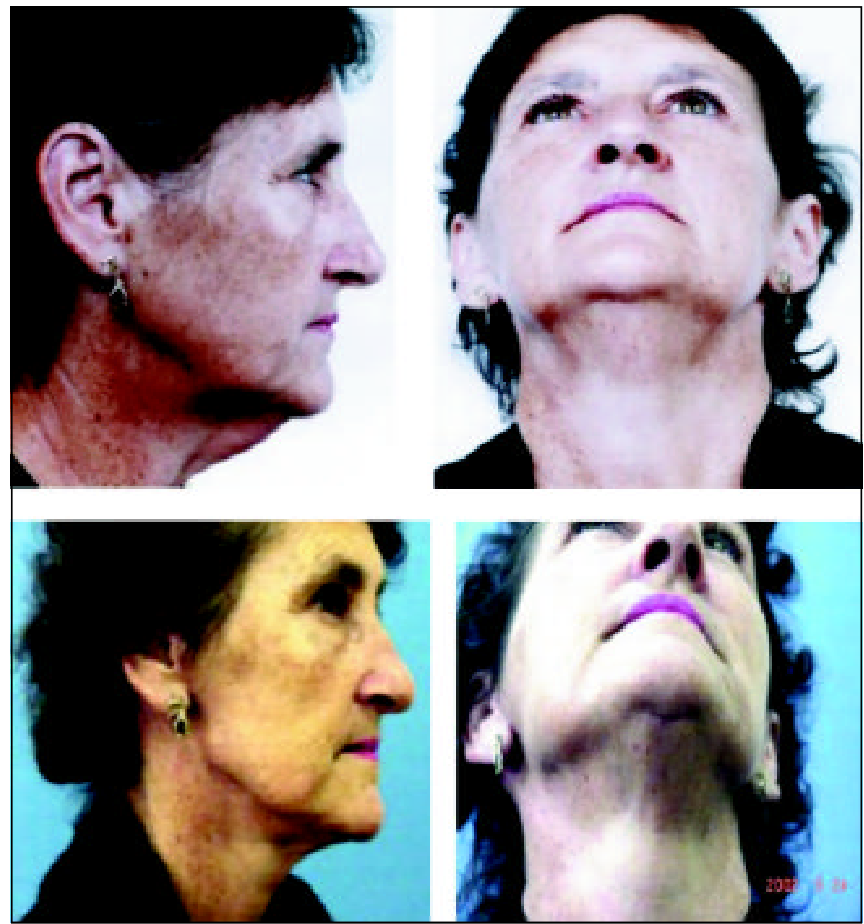

Figura 4. Visão frontal (acima, direita) e lateral (acima, esquerda) de paciente de 58 anos, feminino, com excesso de gordura submental, poucas bandas platismais, ângulo cervicomental obtuso e pouca frouxidão de pele. Pós-operatório ( 60 mês) frontal (abaixo, direita) e lateral (abaixo, esquerda) de cervicoplastia anterior demonstrando regular linha mandibular, ângulo cenvicomental adequado, ausência das bandas platismais e frouxidão da pele e boa proporção do terço inferior da face. 
A cirurgia revisional foi necessária em um paciente que se referia pouca melhora com a cirurgia, sendo realizada com retirada de mais tecido subcutâneo com resultado satisfatório.

Houve complicação em 2 casos. Um paciente apresentou um discreto serohematoma que requereu drenagem e outro paciente apresentou uma cicatriz um pouco alargada. Não houve complicações como formação de quelóides, hipo ou hiperpigmentação, granuloma na incisão, paralisia do m. platisma e necrose de pele.

\section{DISCUSSÃO}

$0 \mathrm{~m}$. platisma é um músculo fino e chato que concorre na expressão facial, e varia no tamanho, espessura e elasticidade. Tem relação estreita com mandíbula, SMAS (sistema músculo-aponeurótico superficial), $m$. peitoral e $m$. deltóide. Pela sua ação, a pele do pescoço se enruga e o canto da boca é tracionado inferiormente, dando uma expressão de repugnância. Na maioria dos casos suas fibras se imbricam na linha média, sob o mento, na região anterior do

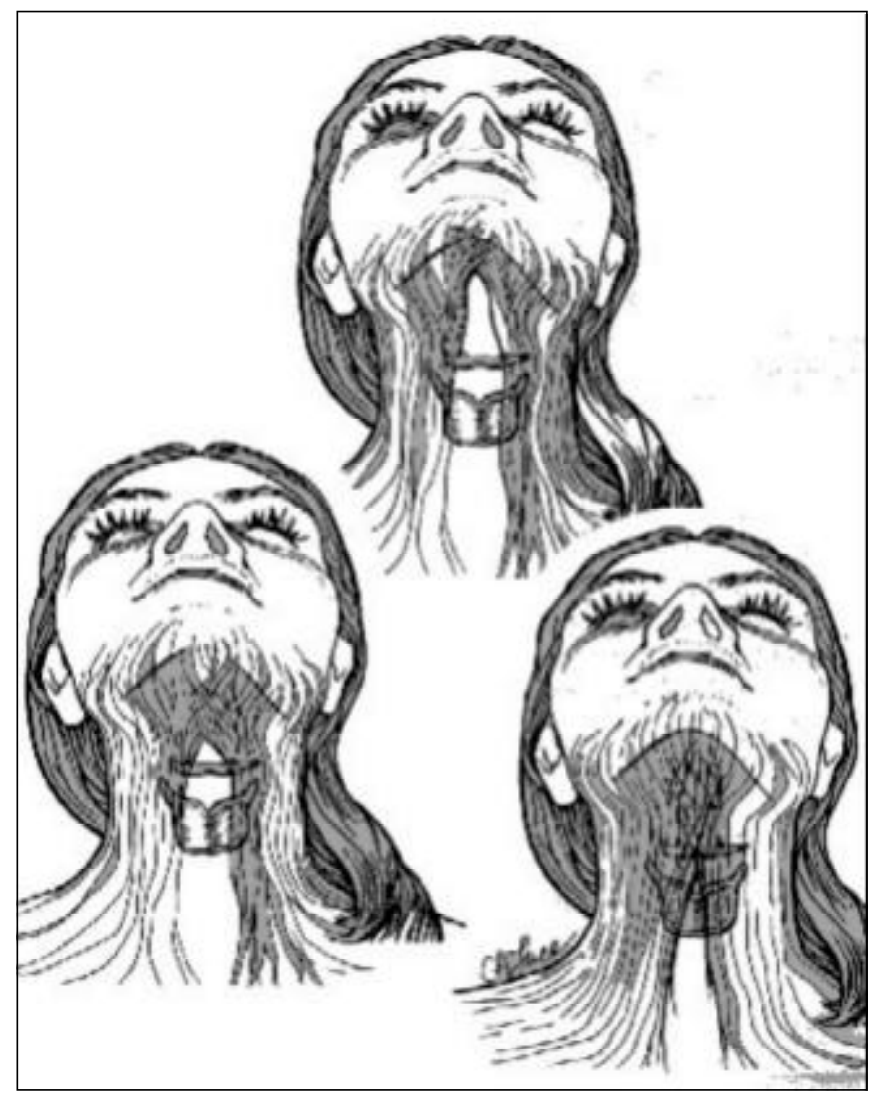

Figura 5. Figura demonstrando os tipos de conformação do músculo platisma quanto à sua imbricação na linha média. pescoço. Em 10 a 15\% dos indivíduos isto não acontece, o que talvez favoreceria a formação das bandas com a chegada dos 40 anos de idade ${ }^{12}$ (Figura 5).

A ação sobre $0 \mathrm{~m}$. platisma vai depender da anatomia do músculo, da mandíbula e do pescoço, da posição do osso hióide, do tamanho da cartilagem tireóide e da quantidade de gordura.

$0 \mathrm{~m}$. platisma pode ser trabalhado de diferentes maneiras, dependendo das alterações apresentadas: a) Elevação e avançamento céfalo-posterior sem excisão ou alteração da linha média; b) Retalho em "L" com excisão parcial lateral; c) Excisão parcial lateral, plicatura e secção medial; d) Excisão total e plicatura medial.

A excisão parcial lateral do m. platisma é indicada quando há indefinição da linha posterior da mandíbula, em pescoços moderadamente magros e com frouxidão leve ou moderada do m. platisma. A excisão ampla do m. platisma, $6 \mathrm{~cm}$ abaixo do ângulo da mandíbula até a cartilagem tireóide, tem indicação em casos de mandíbula estreita, pescoço grosso e face gorda.

A intervenção pode ser medial, pela cervicoplastia anterior como descrevemos, lateral ou ambas simultaneamente. A técnica de acesso medial ao m. platisma consiste numa lipectomia, ressecção vertical das bandas redundantes mediais e plicatura medial ${ }^{13,14}$.

A cervicoplastia anterior é um procedimento acessório simples que pode ser ou não associado ao lifting e deve fazer parte do nosso armamentário cirúrgico. Nos casos com indicação, pode ser associada ao lifting e melhorar consideravelmente a cirurgia, pois a tendência hoje é combinar duas ou mais técnicas para conseguir um resultado final mais duradouro. As razões mais comuns que levam os pacientes a buscarem correções plásticas são a tentativa de melhorar as rugas do pescoço e de eliminar a gordura submentoniana, a papada.

Agindo no m. platisma e na gordura da região submentoniana, através de uma pequena incisão sob a região anterior e inferior da mandíbula, obtém-se um efeito estético adjuvante considerável. Esta ação vai depender da quantidade de gordura submental e submandibular, das bandas do m. platisma e do ângulo cervicomental ${ }^{15}$.

Os pacientes selecionados para a utilização desta técnica isoladamente devem ter boa tonicidade da pele, com leve ou moderada quantidade de gordura.

É indicada em pessoas jovens, com ângulo cervicomental obtuso, pescoço grosso e com gordura. Pode ser ainda indicada em: pacientes de meia-idade ou idosos, com pele elástica, pouca gordura e pouca banda de platisma; indivíduos que já fizeram a cervicoplastia tradicional e persistem sem ângulo cervicomental; pessoas que já se submeteram à lipoaspiração da região com resultado pobre; casos de microgenia leve com indicação de implante. Nas microgenias severas deve se associar à cervicoplastia uma genioplastia ${ }^{10}$. 
A cervicoplastia anterior isoladamente é contraindicada em: frouxidão leve ou ausência de bandas do $\mathrm{m}$. platisma; em ângulo cervicomental normal; em pacientes de meia-idade com bandas grandes de m. platisma e pele sem elasticidade; em velhos com bandas de m. platisma e dobras de pele no pescoço; em "pescoço de touro"; em micrognata com indicação de cirurgia ortognática; em ptose de glândula submandibular ${ }^{10}$.

A cervicoplastia anterior apresenta como vantagens: rapidez, menos trauma, eliminação das bandas, redução da frouxidão, estabilização da tensão da musculatura cervical, aprofundamento do ângulo cervical, não traz problemas de vascularização ao retalho, tem boa aceitação pelos jovens por não apresentar cicatriz posterior e tem menos "aparência de operado"16,17.

A associação da plicatura das bordas anteriores do $\mathrm{m}$. digástrico com Ethybond 2-0 provoca uma contra-tensão do ventre posterior, elevando e posteriorizando o osso hióide. Como os homens apresentam flacidez de pele mais cedo o resultado é melhor em mulheres do que em homens da mesma idade ${ }^{10}$.

Apesar da indicação e do acesso limitado, se usada no paciente certo o resultado é comparável à técnica excisional aberta ${ }^{10,17,18}$.

\section{CONCLUSÕES}

Concluímos que a cervicoplastia, associada ou não à tração lateral pela ritidoplastia é uma técnica que produz resultados satisfatórios na grande maioria dos casos, promovendo melhora do perfil cervical, redução das bandas de platisma e da frouxidão da pele, estabilização da musculatura cervical e acentuação do ângulo cervicomental.

\section{REFERÊNCIASBIBLIOGRÁFICAS}

1. Johnson JB, Hardley RC. The aging face. In: Converse JM, ed. Reconstructive Plastic Surgery. Philadelphia: W.B. Saunders; 1964. p. 1328.

2. Cronin TD, Biggs TM. The Z-plasty for the "turkey globber" neck. Plast Reconstr Surg 1971; 47(6):534-8.

3. Weisman P. A simplified technique in submental lipectomy. Plast Reconstr Surg 1971; 48(5):443-6.

4. Millard DR Jr, Garst WP, Beck RL, Thompson ID. Submental and submandibular lipectomy in conjunction with a face lift, in the male or female. Plast Reconstr Surg 1972; 49(4):385-91.

5. Baker TJ, Gordon HL, Whitlow DR. Our present technique for rhytidectomy. Plast Reconstr Surg 1973; 52(3):232-6.

6. Connell BF. Eyebrow, face, and neck lifts for males. Clin Plast Surg 1978; 5(1):15-28.

7. Gerrero-Santos J. The role of the platysma muscle in rhytidectomy. Clin Plast Surg 1978; 5:29-49.

8. Rees TD. Face-Lift. In: Rees TD, Wood-Smith D, eds. Cosmetic and Facial Surgery. Philadelphia: W.B. Saunders; 1973. p. 203.

9. Feldman JJ. Corset platymaplasty. Plast Reconstr Surg 1990; 85:333-43.

10. Ramirez OM. Cervicoplasty: nonexcisional anterior approach. Plast Reconstr Surg 1997; 99(6):1576-85.

11. Patrocínio JA, Patrocínio LG. Cervicoplastia Anterior. In: Stamm A (editor). Rhinology 2002. Campinas: Komedi; 2002. p. 321-4.

12. Fehrenbach MJ, Herring SW. Músculos. In: Fehrenbach MJ, Herring SW, eds. Anatomia Ilustrada de Cabeça e Pescoço. Bela Vista: Manole; 1986. p. 103-6.

13. Mangat DS, Mcollough EG. Rhytidectomy. In: Cummings CW, ed. Otolaryngology Head and Neck Surgery. St. Louis: Mosby; 1986. p. 409-27.

14. Maniglia JJ. Cirurgia do Rejuvenescimento Facial. In: Colombini $N$, ed. Cirurgia da Face: Interpretação Funcional e Estética. São Paulo: Revinter; 2001. p. 1253-71.

15. Kamer FM, Pieper PG. Facelift: a facial plastic surgeon's perspective. In: Romo III T, Millman AL, eds. Aesthetic Facial Plastic Surgery. New York: Thieme Medical Publishers; 2000. p. 171-87.

16. Keller GS, Cray JE. Percutaneous video-endoscopic necklift with suture suspension platysmaplasty. In: Keller GS, ed. Endocopic Facial Plastic Surgery. St. Louis: Mosby; 1997. p. 137-56.

17. Knize DM. Limited incision submental lipectomy and platysmaplasty. Plast Reconstr Surg 1998; 101(2):473-81.

18. Del Campo AF. Midline platysma muscular overlap for neck restoration. Plast Reconstr Surg 1998; 102(5):1710-4. 